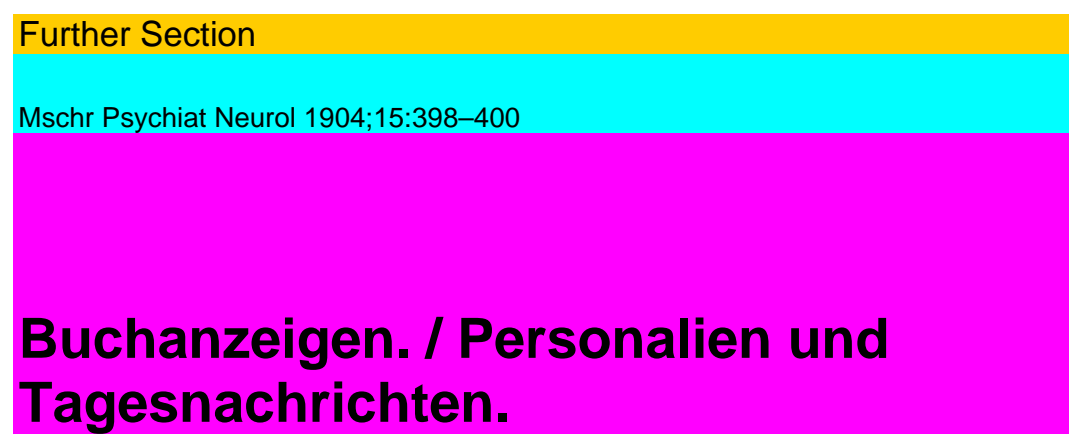

v. Sarbo , Arthur. Der Achillessehnenreflex und seine klinische Bedeutung. Berlin 1903. S. Karger.

Die bis heute noch vielumstrittene Bedeutung des Achillessehnen-

reflexes als diagnostisches Merkmal sucht Verf. an einem umfangreichen Material (302 Gesunden und 884 Nervenkranken) klarzustellen. Er kommt zu dem Ergebnis, dass dem Achillessehnenreflex eine ahnliehe klinische Bedeutung zuzuerkennen ist wie dem Patellarreflex. da jener wie dieser beim Gesunden stets vorhanden, bei degenerativer Erkrankung des peripheren sensiblen wie motorischen Neurons aufgehoben ist.- Da der Achilles sehnenreflex in einer nicht geringen Zahl von Tabe's- und Paralysefallen friiher verschwindet als der Patellarreflex, so isfrrer fur die Friihdiagnose dieser Zustande von wesentlicher Bedeutung. Neumann-Karlsruhe.

Levy, P. E. Die natfirliche Willensbild ung. Eine praktische Anleitung zur geistigen Heilkunde und zur Selbsterziehung. Leipzig 1903. E. Voigtlander

Das vorliegende Werk giebt in popularer Darstellung eine Anleitung durch Autosuggestion den Willen zu erziehen und die Fahigkeit zu erlernen, sich fiber mancherlei subjektive Unpasslichkeiten hinwegzusetzen. Das Buch bringt zweifellos manchen guten Gedanken; Bef. mochte indessen die Anwendung der Autosuggestion fur weniger einfach, ihre Wirkung in allgemeinen fur weniger weitgehend halten.

$\mathrm{G}$ eist-Zschadrass.

Bourneville. Becherches cliniq[ues et therapeutiques sur l'epilepsie, l'hystfirie et l'idiotie. Herausgegeben unter der Mitarbeit mehrerer Assistenten. Paris 1901.

Der 21. Jahresbericht tiber den arztlichen Dienst im Bicetre, der grossen Pariser Krankenanstalt fur idiotische, epileptische und znriick-gebliebene Kinder, reiht sich seinen Vorgangern wiirdig an. Wir greifen aus dem reichon Inhalt die Notiz heraus, dass fiir ilrztliche Besucher des Instituts die Verwaltung eine besonders eingehende Demonstration aller Zweige des Unterrichts und der JKrankenpflege vorgesehen hat. Bourne-ville bittet solche Besuche am Sonnabend Vormittag abzustatten, fiir welche Zeit alle Vorbereitungen getroffen sind.

Interessant ist auch zu ersehen, dass die Einrichtung besonderer Hilfsklassen fiir schwachbegabte Schuler, welche in Deutschland schon in einer Eeihe von St\&dten verwirklicht ist und zwar im wesentlichen durch die Bemuhungen -weitsichtiger Padagogen, jetzt auch fiir JFrankreich von Bourneville und gleichgesinnten Aerzten erstrebt wird.

Unter den 19 wissenschaftlichen Studien, welche der zweite Teil des Bandes enthalt, befinden sich eine Eeihe sorgfaltiger anatomischer uud histologischer Untersuchungen von Idiotengehirnen und -schadeln.

Auf den wissenschaftlichen Teil werden wir noch eingehender in

dieser Monatsschrift im zweiten Bericht fiber Epilepsia und Epileptikerfiirsorge zurfickkommen. 
Liebmann, Alb. Stottemde Kinder. (Sammlung von Abhandlungen aus dem Gebiete der padagogischen Psychologic und Psysiologie von Ziegler und Zi eh en, VI. 2.). Berlin 1903. Reuthe'r u. Reichard.

Die funklionellen kindlichen Spracbstorungen sind bedauerlicher Weise fur die fiberwiegend grosse Mehrzahl der Aerzte wie der Lebrer eine vollige terra incognita. Arbeiten aus sachkundiger Feder, wie die vorliegende, sollten viel mehr gelesen werden, als dies bisher geschieht.

DieL 'scbe Arbeit zeichnet sich vor so vielenkasuistischen Publikationen vorteilhaft dadurch aus, dass jeder einzelne der dargestellten KrankheitsfMJle lehrrfiicb. ist als Paradigma und zugleich interessant durch seine Eigenart. Das Wesen der Sprachhemmung, die das Stottern ausmaeht, sieht L. in der „TJebertreibung des konsonantischen Elements", die Ursache in ererbter oder erworbener nervoser Disposition. Ist die Sprachhemmung in ihren Aufangen erst einmal da, dann entwickelt sie sich zum aus-gesprochenen Stottern vorwiegend aui rein psychischem Wege. Erzieher und Lehrer tragen gar oft durch unzweckmassige Vorschiiften und Uebungen oder durch unangebrachten Tadel und Spott sehr wesentlich dazu bei, dass sich das im Keime vorhandene TJebel vol] entwickelt.

Aus seiner Auffassung vom Wesen des Leidens deduziert L. seine Therapie. Das Prinzip derselben ist Zuriickdrangen des konsonantischen Elements durch Dehnung der Vokale und Bekampfung der Sp)echangst durch JHebung des Selbstvertrauens auf suggestivem Wege. .

Naheres moge der hSchst lehrreichen Arbeit selbst entnommen werden.

Neumann-Karlsruhe.

Taschenkalender fiir Nerven- und Irren-Aerzte 1903. Herausgegeben vonDr. Hans Kurella inBreslau. Berlin, Vogel und Kreienbrink. Der Kalender hat sich seit seinem ersten Erscheinen rasch eingebiirgert und ist den Eachgenossen zu empfehleD. Er enthS.lt diesmal als neuen Zusatz ein Verzeichnis der Heilanstalten und Kurorte, wahrend der therapeutische Teil vermehrt und verbessert worden ist. Das Format ist handlich. Wi ndscheid-Leipzig.

Schreber, Daniel Paul, Denkwiii'digkeiten eines Nervenkranken nebst NachtrS,gen und einem Anhang tiber die Frage: „Unter welch en Voraussetzungen darf eine fur geisteskrank erachtete Person gegen ihren Willen in einer Heilanstalt festgehalten werden?" Leipzig 19,03. Oswald Mutze.

Der Titel des Buches wird bei der Bedeutung, die neuerdings die Frage von der sogen. unberechtigten Internierung von Geisteskranken erlangt hat, gewiss manchen zur Lekture des Werkes veranlassen. Was in ihm der Nichtmediziner sucht und findet, kann dem Fachmann gleichgiltig sein. Ob der Laie ein Wares Drteil sich iiber den vorliegenden Fall bilden, speziell die Frage, ob der Verf. tatsachlich mit Enrecht auf dem Sonnenstein festgehalten worden ist, entscheiden kann, muss indessen sehr bezweifelt werden, und von diesem Gesichtspunkte aus ist die VerSffentlichung der Denkwiirdigkeiten doch mit grosser Vorsicht aufzunehmen. Wird doch mancher, der nicht imstande ist, sich ein einwandsfreies Urteil fiber das Buch zu bilden, zu sehr lalschen Vorstellungen gelangen. Was aber das Buch fur den Fachmann wertvoll macht - und das wird wohl gerade der Verf. am allerwenigsten beabsichtigt haben! — das ist die Darstellung der Symptome einer Paranoia, wie sie wohl bisher in dieser VollstSndigkeit und diesem TJmfange noch nicht geboten worden ist. Der

Verf., ein ausserst scharfsinniger Jurist, versteht es in ausgezeichneter Weise, seine im Beruf ihm zur Gewohnheit gewordene Logik auf die Erzeugnisse seiner kranken Phantasie zu iibertragen und bietet auf diese Art eine Darstellung, die der kundige Arzt nur als vollendet bezeichnen kann. In diesem Sinne mag das Buch den Fachgenossen zum Studium empfohlen sein. Ob seine Veroffentlichung notwendig war, dariiber kann man allerdings sehr geteilter Meinung sein! In die .iuristischen Probleme einzugehen, speziell in die Diskussion fiber die Berechtigung des Gerichts, die Wi ndscheid- Leipzig. Entmfindigung aufzuLeben, ist hier nicht der Ort. 
Personalien und Tagesnachrichten.

Forel, A., u. Mahsim, A. T., Crime et a nomalies mentales constituti on elles. Genf 1902. Henry Kfindig.

Im vorliegenden Buche. $\mathrm{W}<$ lches Forel in Gemeinschaft mit Prof.

Mahaim-Lausanne veroffentlicht, tritt er fiir die Errichtung besonderer

Anstalten fiir vermindert Zurechnnngsfahige ein. Er weist nach, dass die heutigen Verhaltnisse unhaltbar sind, wo die psychopathischen Gewohnheits-Verbrecher zwischen Strafanstalt, Irrenanstalt und Korrektionshaus ziellos hin- und hergeschoben werden, weil sie in keine dieser Anstalten passen, immer von neuem in Ereibeit gesetzt werden und aufs neue straffallig werden. Die vorgeschlagenen Asyle sollen unter- die Oberaufsicht einer Kommissiou von Irrenarzten, Juristen und Stralanstaltsdirektoren gestellt werden und einen Psychiater als Direktor erbalten. Sie sind fern von der Stadt auf dem Lande zu errichten und zwar in Pavillonsystem. wodurch Individualisierung und eventuelle Erweiterungsbauten erleichtert werden. Die Internierten sind mit Feldarbeit und in Werkstatten zu beschaftigen. - Das kasuistische Material, welches Verf. in der ersten Halfte des Buches bringt, wird psychiatrische und juristische Kreise in gleicher Weise interessieren.

Bolte-Bremen.

\section{Personalien und Tagesnachrichten.}

Hermann Emminghaus f. Am 17. Eebruar starb Prof. Hermann Emminghaus im Alter von fast 60 Jahren nach langerem Siechtum. E. habilitierte sich im Jahre 1873 zu Wiirzburg. 1880 folgte er einem Ruf nach Dorpat. Seat 1887 leitete er die psychiatrische Klinik in Ereiburg. Im Herbst 1902 erbat er wegen eines schweren Leidens seine Pensionierung. Seine beiden Hauptwerke sind die „Allgemeine Psychopathologie" aus dem Jalve 1878 und „Die psychischen StŠrungen des Kindesalters" aus dem Jahre 1887. Namentlich das letztere Werk ist fiir das Studium der Kinderpsychosen grundlegend geworden. In dasselbe Gebiet gehOren seine bekannten Beitrage in Maschka's Handbuch der gerichtlichen Medizin. Seine letzte gr8ssere Arbeit war der Beitrag zum Penzoldt-Stintzing'schen Handbuch. „Behand-lung des Irreseins im allgemeinen" (2. Aufl. 1898). Unter den alteren Werken seien namentlich noch die Abhandlungen uber Lyssa und epidemi-sche Meningitis genannt. Eine bewunderungswiirdige Literaturkenntnis und GrCndlichkeit zeichnet alle Arbeiten von Emminghaus aus. In der Geschichte der Psychiatrie nimmt er eine hervorragende Stelle ein.

An Stelle von Prof. Bonhoeffer wurde PrivatdozentDr. E. Meyer aus Kiel als Professor der Psychiatrie und Nervenkrankheiten nach KSnigs-berg berufen.

Privat-Dozent Dr. Hugo Liepmann in Berlin ist zum Professor ernannt worden.

Anlasslich des Hinscheidens von Prof. Bum in ist seine Schrift „Zur Geschichte der panoptischen Irrenansfcalten" neugedruckt worden und wird von der Direktion der Kreisirrenanstalt Erlangen versendet (a M. 1.-).

Unser verehrter Mitarbeiter Dr. Fri'edlander hat ein Kurhaus ${ }^{1}$ fiir Nervenkranke und Kranksinnige auf der Hohen Mark im Taunus erSfinet.

Der 14. Kongress der Irren- und Nervenarzte Frankreichs findet am 1.-7. August in Pau statt. Deny wird tiber die Demences vesaniques, Sano tiber die Localisations motrices dans la moelle, K6raval fiber Mesures a prendre contre les alidn $\wedge$ s criminels referieren. 